\title{
Adaptive Slope Voltage Control for Distributed Generation Inverters with Improved Transient Performance
}

\author{
Juan M. Rey, Miguel Castilla, Jaume Miret, Member, IEEE, Antonio Camacho, and Ramon Guzman
}

\begin{abstract}
Reactive power injection in distributed generation inverters is an useful ancillary service for grid supporting purposes. For grid-feeding converters, the slope control method is the most common voltage regulation strategy used in local (communication-less) applications. Despite its simplicity, this method offers limited dynamic properties in scenarios with changing operation conditions. In this sense, this paper presents an adaptive slope voltage control which provides an improved transient performance against operating variations. To derive the control configuration, a control-oriented mathematical model is developed. The accuracy of the modeling and the performance of the proposed control are validated by selected experimental results.
\end{abstract}

Index Terms-Adaptive slope voltage, distributed generation, grid-feeding power converter, slope voltage control, voltage regulation.

\section{INTRODUCTION}

$\mathbf{I}$ $\mathrm{N}$ recent years, distributed generation based on renewable energy sources has gained more attention because of evergrowing concerns on environmental issues and energy costs [1]. This has led to an increase in the number of renewablebased distributed generators (DGs) connected to the electrical network [2]. The connection of a large amount of DGs can cause voltage deviations and impairments in the stability of the grid [3]. For this reason, grid codes require that DGs provide ancillary services aimed to mitigate these adverse effects.

To connect DGs to the electrical grid, three-phase power converters are employed as interfaces. Depending on their operation aspects, converters can be classified into grid-forming and grid-feeding [4], [5]. Grid-forming power converters are controlled with the main objective of regulating its output voltage. Because of this, its operation can be modeled using a power-controlled voltage source. A common method to achieve the voltage regulation consists of including control loops in which the frequency and amplitude of the voltage follow references that are corrected by terms proportional to

This work was supported by the Ministry of Economy and Competitiveness of Spain and by the European Regional Development Fund under project ENE2015-64087-C2-1-R.

Juan M. Rey is with Escuela de Ingenierías Eléctrica, Electrónica y de Telecomunicaciones (E3T), Universidad Industrial de Santander (UIS), Bucaramanga 680002, Colombia (e-mail: juanmrey@uis.edu.co).

M. Castilla and J. Miret are with the Department of Electronic Engineering, Technical University of Catalonia, Vilanova i la Geltrú 08800, Spain (e-mail: miquel.castilla@upc.edu; jmiret@eel.upc.edu)

A. Camacho and R. Guzman are with the Department of Automatic Control, Technical University of Catalonia, Vilanova i la Geltrú 08800, Spain (email: camachem@gmail.com; ramon.guzman@upc.edu). the active and reactive powers, respectively. For this type of converters, this control strategy is usually called droop method [6], [7]. This type of control is commonly framed in hierarchical controls, which are characterized by grouping control objectives according through different speeds of response [8].

Grid-feeding power converters are controlled with the main objective of injecting active and reactive power following determined references. Due to this, its operation can be modeled using a power-controlled current source. Particularly, reactive power injection can be controlled as an ancillary service for grid supporting purposes. It can be useful for voltage regulation in several scenarios including load changes and abnormal grid conditions [9]-[11].

One of the most relevant applications of the grid-feeding power converters are the wind generators [12]. Due to the fast growing number of this type of DGs, grid codes are including stringent requirements related to voltage support at the point of connection [13]. A common strategy to achieve an effective voltage regulation consists of implement a control loop in which the voltage amplitude follows a reference that is corrected by a term proportional to the injected/absorbed reactive power. For grid-feeding converters, this control strategy is usually called slope method [14]-[16]. This control approach is widely used for its simplicity and flexibility. The slope method can be implemented according to different control schemes, most of them based on static transfer functions using strategies such as proportional, proportional-integral and integral with proportional feedback controllers [17]-[20]. Even though these control strategies are suitable alternatives to regulate the voltage, in this paper an analysis based on the modeling proposals presented in [21] and [22] has been made, identifying that the static slope voltage control offers limited dynamic properties in scenarios where the operation conditions are changing. This drawback risks the operation of the DG when is implemented under hierarchical controllers and the fulfillment of the grid codes requirements.

Thus, considering this limitation, in this paper an adaptive slope voltage control for DGs is presented. Compared with static approaches, this proposal provides an improved transient performance against grid operating variations, guaranteeing a constant settling time. This control is based on an adaptive gain which is updated in real-time according to an estimation of the grid impedance. To derive the control configuration, a controloriented mathematical model is obtained from the static slope voltage control. The model is also used to propose a design procedure for the control parameters. 
The paper is organized as follows. Section II presents a general description of the system. Section III develops the steady-state, small-signal and closed-loop modeling. In Section IV, the proposed control is presented including the control architecture and the design consideration. Experimental results to validate the proposal are presented and discussed in Section V. Finally, general conclusions are drawn in Section VI.

\section{SySTEM DESCRIPTION}

This Section presents the system description including general concepts about grid-connected DG modeling, reactive power injection and slope voltage control.

\section{A. Grid-connected Distributed Generation Systems}

The diagram of a grid-connected DG system is presented in Fig. 1. The primary power source is connected to a three-phase inverter. Between them two, a dc-link is needed to balance the power flow from the source to the grid. The inverter uses an LCL filter (composed by $L_{i}, C$ and $L_{o}$ ) in order to reject switching noise and harmonics. The connection to the grid is done at the point of common coupling (PCC). The grid is assumed mostly inductive and is modeled by a voltage source and an equivalent grid impedance $L_{g}$.

The main task of a grid-feeding power inverter is to deliver the generated active power to the grid by controlling the amount of injected current. In order to extract the maximum power from the power source, the voltage at the dc-link $v_{d c}$ is regulated by an external voltage controller, which provides the active power reference $P^{*}$ that should be injected [23].

Besides the active power injection, inverters can supply reactive power to the grid following grid codes requirements [24], [25]. The range of variation of the voltage at the PCC caused by this injection depends on the inverter current capacity and the grid inductance. A properly generated reactive power reference $Q^{*}$ can be useful to keep the PCC voltage around the nominal values and avoid exceeding the limits of operation imposed by the grid code. Different control approaches can be implemented to generate $Q^{*}$. The reactive power injection technique used in this work is discussed in the next subsection.

Once the reference $Q^{*}$ is defined, a current reference $i^{*}$ is calculated, which is followed by the controller. This reference is generated as follows

$$
i^{*}=\frac{P^{*} v}{V^{2}}+\frac{Q^{*} v^{\perp}}{V^{2}} .
$$

where $v^{\perp}$ denotes the quadrature of the PCC voltage $v$ and $V$ the amplitude of $v$ [26], [27]. The current reference $i^{*}$ is used in the current loop and the space-vector modulator (SVM) to control the power switches of the inverter [28].

\section{B. Local Reactive Power Injection Techniques}

There are several local control techniques for the injection of reactive power in order to regulate the PCC voltage. They can be grouped in three general forms:

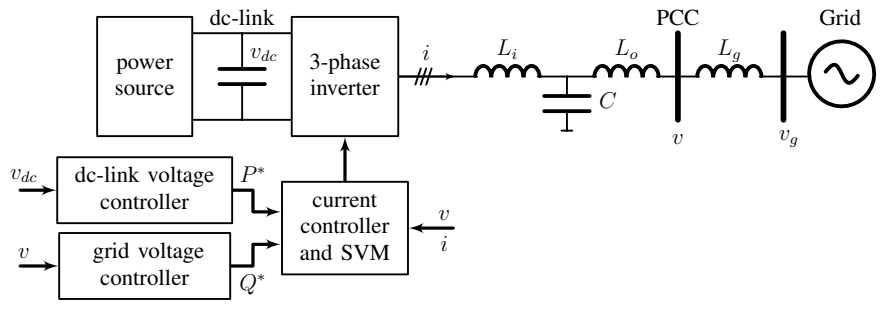

Figure 1. Circuit diagram of a grid-connected DG system.

1) Constant reactive power reference: This method forces to follow a constant reactive power reference $\left(Q=Q^{*}\right)$ received from a higher hierarchical control level [2].

2) Constant voltage regulation: It is based on a local voltage control that regulates the PCC voltage amplitude to a reference value $\left(V=V^{*}\right)$ [29].

3) Slope voltage control: This last method regulates the voltage amplitude proportionally to the reactive power injection [14]-[16].

Fig. 2 illustrates the operation of these control strategies. The vertical axis shows the PCC voltage amplitude $V$ and the horizontal axis the injected reactive power $Q$. In each graph, the characteristic curve of the system is displayed in three different grid voltage scenarios (solid lines). The values over the vertical axis, $V_{g_{H}}, V_{g_{N}}$ and $V_{g_{L}}$ are voltage amplitudes when $Q$ is not injected. Particularly, $V_{g_{N}}$ is the voltage amplitude for nominal conditions, and $V_{g_{H}}$ and $V_{g_{L}}$ the maximum and minimum values expected. The slope of the lines depends on the value of $L_{g}$. The operation points of the system are represented by the intersections between the control law (dashed line) and the characteristic curves.

The performance of the first control technique is depicted in Fig. 2(a). As the amount of reactive power injected $Q^{*}$ is fixed, the PCC voltage increases proportionally to the grid voltage and no voltage regulation is performed.

Conversely, the second control technique provides accurate voltage regulation, as it can be seen in Fig. 2(b). However, the range of reactive power required to satisfy the objective $V=V^{*}$ is very large considering the differences between the operation points related to $V_{g H}$ and $V_{g L}$ (red line and yellow line). Therefore, depending on the power converter capacity, the control objective could not be reached.

Finally, in the third control technique, the injection of $Q$ generates a linear decrease in the voltage, as it can be seen in Fig. 2(c). A lower range of variations on $Q$ allows obtaining different operation points according to the selected slope and voltage reference $V^{*}$. Notice that this last variable works as a control parameter and not as a control objective, since the nature of the control technique produces a stationary error between $V^{*}$ and $V$.

\section{Slope Voltage Control}

Fig. 3(a) depicts the proportional-based scheme, the simplest implementation of a slope voltage control. The exclusive use of a proportional term does not offer the possibility to adjust the dynamic response of the system. This feature can be achieved by adding an integral term to constitute 


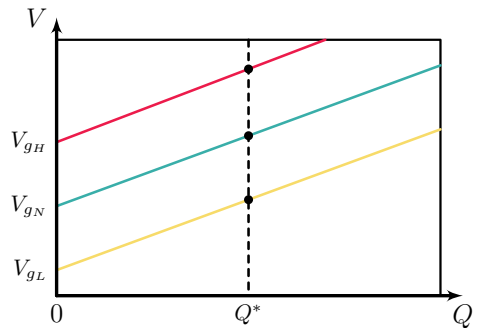

(a)

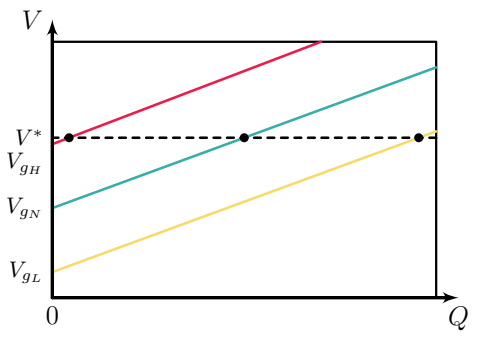

(b)

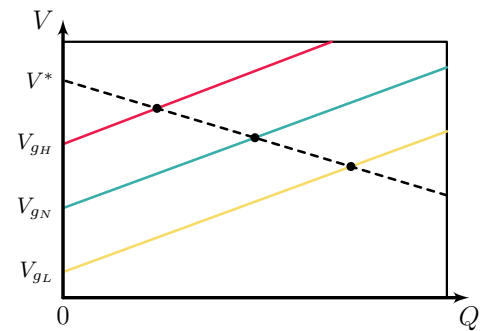

(c)

Figure 2. Reactive power injection methods: (a) constant reactive power reference, (b) constant voltage regulation, (c) slope voltage regulation.

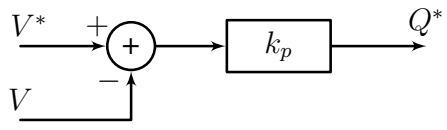

(a)

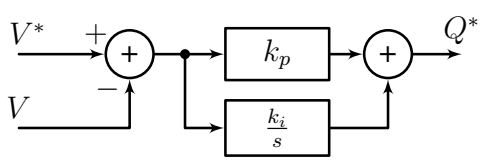

(b)

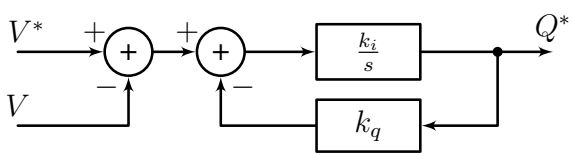

(c)

Figure 3. Slope voltage control schemes: (a) proportional, (b) proportionalintegral, (c) integral with proportional feedback.

a proportional-integral scheme, as shown in Fig. 3(b). This configuration allows to adjust the dynamic response by the proper design of the control parameters. However, as discussed in [30], in scenarios in which multiple inverters operate in parallel, the stability of proportional-integral schemes becomes a critical issue. Typically, this configuration is very sensitive to the accuracy of the measurements and for this reason, any slightest measurement error will be integrated causing hunting phenomena, the cumulative integration of small measurement errors in distributed systems, leading to the instability [31].

An alternative for this is the integral with proportional feedback-based scheme, depicted in Fig. 3(c). This configuration provides an adjustable dynamic response with a degraded voltage regulation, as described above [15].

The control law of this scheme can be expressed in Laplace domain as

$$
Q^{*}=\frac{k_{i}}{s+k_{i} k_{q}}\left(V^{*}-V\right)
$$

where $k_{i}$ is the gain of the integral controller, $k_{q}$ is the proportional feedback gain that provides the slope characteristic and $s$ is the Laplace operator.

Notice that the control law in (2) indicates that this configuration behaves as a proportional control with a low-pass filter. This means that although the dynamic response can be adjustable by a proper control parameters design, this alternative does not suffer from the critical instability issues of the proportional-integral distributed systems.

In the next Section, the modeling of the system is presented with the aim of analyzing its characteristics and limitations.

\section{SYSTEM MODELING}

The system modeling is based on the averaging approach presented in [21]. This modeling contains the information of the dominant poles related to the slow time-varying variables of the system (for this reason, the dynamics related to the inner loops are not considered). Using it as a starting point, the steady-state expression that determines the operating point is obtained. Around this point, the small-signal model is derived and the control transfer function presented. Once these expressions are obtained, the characteristics and limitations of the static slope voltage control are discussed.

\section{A. Modeling of the Grid-connected DG System}

The diagram presented in Fig. 4 represents a DG connected to the grid. As previously discussed, the DG behaves as a power-controlled current source, which exports the active and reactive power references $P^{*}$ and $Q^{*}$. The differential equation that relates the PCC and grid voltages can be expressed as

$$
L_{g} \frac{d i}{d t}=v-v_{g}
$$

where

$$
\begin{gathered}
v=V \cos (\omega t) \\
v_{g}=V_{g} \cos (\omega t+\phi) .
\end{gathered}
$$

Without loss of generality, the phase angle of the PCC voltage is considered to be zero and the phase between the PCC and the grid is $\phi$.

A perfect tracking between the reference current and the injected current is assumed. Therefore, from (1) and (4), the injected current can be expressed as

$$
i=\frac{2}{3} \frac{P^{*}}{V} \cos (\omega t)+\frac{2}{3} \frac{Q^{*}}{V} \sin (\omega t) .
$$

The main objective of the slope voltage control is to roughly regulate the PCC voltage amplitude, which is a slow timevarying variable. Thus, it is possible to derive a low-frequency model using the procedure described in [21]. By inserting (4), (5) and (6) in (3) and applying the aforementioned 


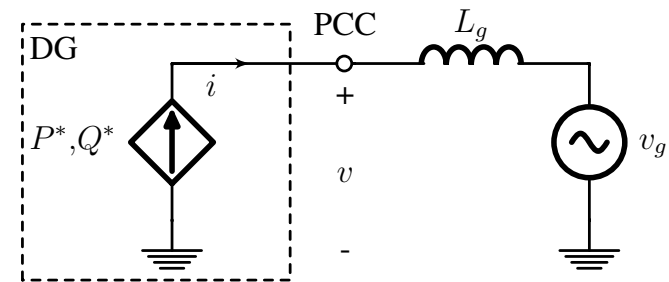

Figure 4. Circuit model of the grid-connected DG.

modeling procedure, the simplified averaged model of the gridconnected DG is obtained as

$$
V_{g}=V-\frac{2 L_{g}}{3 V}\left(Q^{*} \omega+\frac{d P^{*}}{d t}-\frac{P^{*}}{V} \frac{d V}{d t}\right) .
$$

Further details of the derivation of (7) are given in Appendix A. From this non-linear expression, the steady-state and smallsignal models of the power system can be derived as presented in the following subsection.

\section{B. Steady-State and Small-Signal Modeling}

In order to extract the equivalent dynamic model of the power converter, small-signal theory is employed. The derivation of the main equations is obtained according to the procedure presented in [22]. The variables of interest of the small-signal analysis are the PCC voltage amplitude $V$ and the injected reactive power $Q^{*}$. Then, they can be expressed decomposed in quiescent values and small-signal variables as

$$
\begin{aligned}
V & =\bar{V}+\hat{v} \\
Q^{*} & =\bar{Q}^{*}+\hat{q}^{*}
\end{aligned}
$$

where the variables with superscript ${ }^{-}$denote the steady-state values and the superscript ^ identifies the small-signal variations. The other variables in (7) are external disturbances and, then, they do not affect the system stability. For this reason, the small-signal variations are omitted in these variables

$$
\begin{aligned}
V_{g} & =\bar{V}_{g} \\
P^{*} & =\bar{P}^{*} .
\end{aligned}
$$

By substituting (8)-(11) into (7), a mathematical model with small-signal and quiescent variables is obtained. Taking the quiescent values separately, the following relationship is deduced

$$
\bar{V}=\bar{V}_{g}+\frac{2}{3} \frac{\omega L_{g}}{\bar{V}_{g}} \bar{Q}^{*} .
$$

As expected, in this expression it is possible to observe how the reactive power can regulate the PCC voltage amplitude. Note also that this regulation is based on a proportional relation according to the value of the grid inductance $L_{g}$.

The small-signal transfer function can be derived by disregarding the steady-state and the second-order small-signal terms [32]. In Laplace domain, the reactive-power-to-voltage transfer function is written as

$$
G(s)=\frac{\hat{v}}{\hat{q}^{*}}=\frac{\omega \bar{V}}{\bar{P}^{*}} \frac{1}{s+\frac{3 \bar{V}\left(2 \bar{V}-\bar{V}_{g}\right)}{2 L_{g} \bar{P}^{*}}} .
$$

Note that this transfer function represents a first order system with a high frequency pole. This is due to the low power of the inverter in relation to the short-circuit power of the installation, which makes it a high-frequency pole even in the worst case scenario, i.e., when the inverter supplies its maximum power; see (13). Using the data of the experimental setup of Section V, it is observed that this pole is more than 5000 times larger than the dominant low-frequency pole of the closed-loop system in all the considered tests. Thus, the reactive-power-to-voltage transfer function (13) can be simplified as

$$
G(s) \simeq \frac{2}{3} \frac{\omega L_{g}}{2 \bar{V}^{-} \bar{V}_{g}}
$$

\section{Closed-Loop Modeling}

Fig. 5 shows the small-signal block diagram of the system. The transfer function of the slope voltage controller $H(s)$ can be rewritten from (2) as follows

$$
H(s)=\frac{\hat{q}^{*}}{\hat{v}^{*}-\hat{v}}=\frac{k_{i}}{s+k_{i} k_{q}} .
$$

Thus, from (14) and (15), the closed-loop transfer function of the system is obtained as

$$
\frac{\hat{v}}{\hat{v}^{*}}(s)=\frac{2}{3} \frac{\omega L_{g}}{2 \bar{V}-\bar{V}_{g}}\left(\frac{k_{i}}{s+k_{i}\left(k_{q}+\frac{2}{3} \frac{\omega L_{g}}{2 \bar{V}-\bar{V}_{g}}\right)}\right) .
$$

Note that the resulting model is a first-order transfer function. Particularly, it is a low-pass filter function with a crossover angular frequency $\omega_{c}$ of

$$
\omega_{c}=k_{i}\left(k_{q}+\frac{2}{3} \frac{\omega L_{g}}{2 \bar{V}-\bar{V}_{g}}\right) .
$$

In the next subsection, the characteristics and limitations of the slope voltage control are discussed, based on the obtained expressions.

\section{Static Slope Voltage Features}

From (2), the control law in steady-state is deduced as

$$
\bar{V}=V^{*}-k_{q} \bar{Q}^{*} .
$$

Note that only the control parameters $V^{*}$ and $k_{q}$ appear in the steady-state solution. From a control design viewpoint, these parameters can be selected to provide a desired steady-state operation.

Once these parameters are set, the dynamics of the control response, which is related with the value of $\omega_{c}$, depends mainly on the selection of $k_{i}$. As shown in (17), variations in the grid impedance affect the dynamics of the control response if $k_{i}$ is assumed as a static parameter. In large power systems, changes in the grid impedance are almost negligible. However, in distributed power systems, the variations in grid impedances are not negligible because they can degrade the design and performance of the DG's control strategies or even compromise the stability under certain circumstances [33]. 


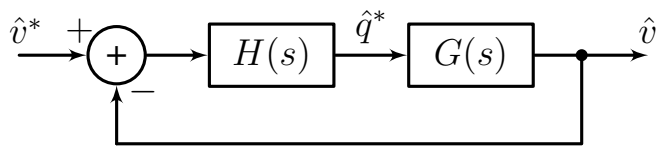

Figure 5. Block diagram of the small-signal model of the grid-connected converter.

The contributions in modeling of grid-feeding inverters presented in [21] and [22] allowed to identify this limitation of the static slope voltage control. Considering this, the next section presents a control proposal that overcomes this feature by introducing an adaptive characteristic. The proposal provides robustness to the transient response to variations in grid operation and, therefore, improves the performance of this type of controller in a simple but effective way that had not previously been presented in the literature.

\section{Proposed Control}

In this section, the proposed control architecture, the grid estimation method and the design considerations are presented.

\section{A. Control Architecture}

The proposed slope voltage control is based on an adaptive integral with proportional feedback-based scheme, similar to the one depicted in Fig. 3(c). However, the parameter $k_{i}$ is replaced by the adaptive gain $k_{i}^{\prime}$ calculated as follows

$$
k_{i}^{\prime}=\frac{\omega_{c}^{\prime}}{\left(k_{q}+\frac{2}{3} \frac{\omega L_{g}(t)}{2 \bar{V}-\bar{V}_{g}}\right)}
$$

where $\omega_{c}^{\prime}$ is the constant cross-over frequency which is responsible to fix the settling time of the system

$$
t s \simeq \frac{5}{\omega_{c}^{\prime}} .
$$

In addition, $L_{g}(t)$ is the real-time estimation of the grid impedance and variables as $V_{g}, V$ and $\omega$ are measured or calculated. The possibility of adapting $k_{i}^{\prime}$ according to measured variables and desired parameters enhances the transient performance against changes in the system operation. Particularly, allows to guarantee a desired settling time even in the presence of grid impedance variations, without affecting the steady-state operation points. This characteristic is favorable in terms of the stability of the DG operation. By controlling the time of response, it is possible to decouple the control strategy from any other layer in the framework of a hierarchical control. Also, as discussed above, this feature can be adapted to different implementation scenarios for the strict fulfillment of the grid codes requirements.

\section{B. Grid Impedance Estimation Method}

The online estimation of the grid impedance is useful for islanding local detection and to guarantee a good performance of adaptive control strategies as the proposed in this work [34]. Grid impedance estimation strategies can be classified in two main groups: passive and active methods. On the one hand, passive methods (non-intrusive) are characterized by estimating the network impedance using measurements already presented in the system, reducing its impact on the grid operation [35]-[37]. These methods offer accurate grid impedance estimations. However, when they are embedded in non-dedicated platforms, its time of estimation can represent a limitation for adaptive control purposes. On the other hand, active methods extract data from the grid injecting systematic perturbations in different frequencies [38]. Once the grid responses are sensed, the information is processed and the grid impedance is estimated using fast algorithms based on techniques as recursive least-square minimization [39] or particle swarm optimization [40], among others. Advances in active methods have allowed a considerable reduction in the time response of estimation and, for this reason, these are the most appropriate methods to be implemented together with the proposed control.

For this study, the method selected for the implementation is the presented in [39]. This method is based on an algorithm introduced by the authors in [41] and [42]. After a preprocessing stage in which the negative sequence is eliminated and the signals are converters to $d q$ rotating synchronous reference frame using a grid synchronization block, the estimation is reduced to a linear regression problem in the complex field. Once at least two measurement points have been gathered, the algorithm finds the best-fit parameters solving a minimization error function. This method offers a light computational charge due to the simplicity of the regression problem leading to accurate and fast estimations.

In general, the control strategy presented in this paper can be implemented with any type of grid impedance estimation method as long as it has an estimation response sufficiently rapid so that the dynamics are not at risk of coupling. As a design guide, it is recommended to select a method with an estimation response time less than a quarter of the expected settling time of the control strategy.

\section{Design Considerations}

The proposed control scheme has three design parameters that determine the steady-state operation point and the dynamics of the control response: $V^{*}, k_{q}$ and $k_{i}$. In the next subsections, design considerations to calculate these parameters are presented. This procedure is based on the previously derived simplified model.

1) Design of Steady-State Control Parameters: In general, the design of the steady-state control parameters is done for determined nominal conditions. In this work, the slope voltage control will be selected in order to follow the behavior shown in Fig. 6, where is expected a nominal operation point with a reactive power injection equal to $Q_{\max } / 2$. The maximum value of reactive power $Q_{\max }$, is dependent on the active power supplied, $Q_{\max }=f(P)$, and can be calculated as

$$
Q_{\max }=\sqrt{S^{2}-P_{\max }^{2}}
$$

where $S$ is the apparent power capacity of the inverter and $P_{\max }$ is the maximum active power production of the power source. Thus, according to Fig. 6 , the reference voltage $V^{*}$ can be set as

$$
V^{*}=V_{\max }
$$




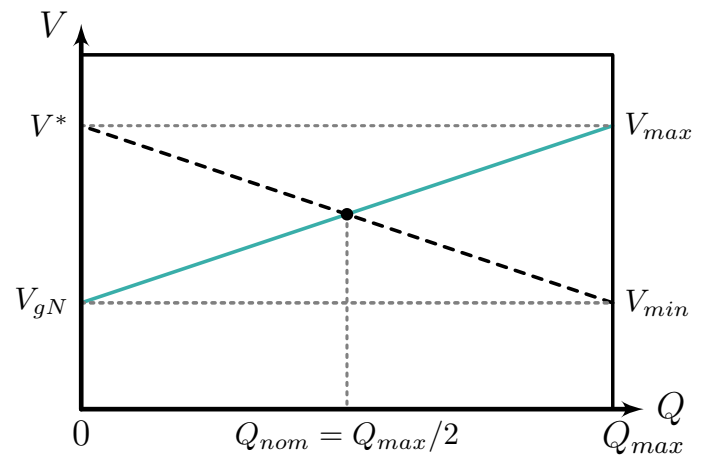

Figure 6. Static diagram showing the desired behavior of the system in steadystate.

and, considering (12), expressed as

$$
V^{*}=V_{\min }+\frac{2}{3} \frac{\omega_{0} L_{g_{0}}}{V_{\min }} Q_{\max }
$$

where $\omega_{0}$ and $L_{g_{0}}$ are the nominal values of frequency and grid impedance, respectively. It is important to highlight that the selection of $V_{\max }$ must be done considering the corresponding grid code guidelines. In this way, it is ensured that the operation of the system will be maintained in the appropriate range. Considering the small variations of the operating frequency, for design purposes $\omega$ is assumed as $\omega_{0}$.

Additionally, the control parameter $k_{q}$ must be selected to guarantee the symmetry shown in Fig. 6. This parameter determines the slope of the control law (in dashed line). To locate the equilibrium point in the middle of the operating range, $k_{q}$ should be calculated as

$$
k_{q}=\frac{\Delta V}{\Delta Q}
$$

where $\Delta V$ is the expected voltage deviation and $\Delta Q$ is the maximum reactive power injection $\left(Q_{\max }\right.$, in this case). Hence, following these design conditions and using (22) and (23), the parameter $k_{q}$ can be calculated as

$$
k_{q}=\frac{V_{\max }-V_{\min }}{Q_{\max }}=\frac{2}{3} \frac{\omega_{0} L_{g_{0}}}{V_{\min }} .
$$

2) Adaptive Control Parameter: As indicated above, $k_{i}^{\prime}$ is a control parameter that depends on $\omega_{c}^{\prime}$. This parameter must be selected at least one decade below the grid frequency because it is desired to: a) avoid interference with the current control loop of the inverter and b) reject harmonics and noise in the grid voltage. Using this design guideline and the grid impedance estimation, a robust transient response is obtained, as verified experimentally in next Section.

\section{EXPERIMENTAL RESUlts}

A low-rated power experimental prototype was built using a 2.24-kVA GUASCH three-phase bridge as power converter, an LCL filter, and inductors modeling the grid, following the scheme presented in Fig. 1. The power source was emulated using an AMREL-SPS-800-12 DC-source and the utility grid by means of a programmable three-phase Pacific AMX-360 AC-source. A Texas Instruments TMS320F28335 floating
Table I

SYSTEM PARAMETERS AND DESIGN SPECIFICATIONS

\begin{tabular}{lcr}
\hline \hline Symbol & Description & Value \\
\hline$S$ & Converter rated power & $2.24 \mathrm{kVA}$ \\
$P_{\max }$ & Maximum active power production & $2 \mathrm{~kW}$ \\
$Q_{\max }$ & Maximum reactive power & $1 \mathrm{kVAr}$ \\
$V_{d c}$ & DC-link voltage & $400 \mathrm{~V}$ \\
$V_{g N}$ & Grid voltage & $110 \sqrt{2} \mathrm{~V}$ \\
$\omega_{0}$ & Grid frequency & $2 \pi 60 \mathrm{~Hz}$ \\
$L_{i}$ & LCL inverter side inductance & $5 \mathrm{mH}$ \\
$C$ & LCL capacitor & $1.5 \mu F$ \\
$L_{o}$ & LCL grid side inductance & $2 \mathrm{mH}$ \\
$L_{g}$ & Nominal grid inductance & $2.5 \mathrm{mH}$ \\
$f_{s}$ & Sampling frequency & $10 \mathrm{kHz}$ \\
$f_{s w}$ & Switching frequency & $10 \mathrm{kHz}$ \\
$\omega_{c}^{\prime}$ & Cross-over angular frequency & $2 \pi \mathrm{rad} / \mathrm{s}$ \\
$V^{*}$ & Voltage reference & $1.026 \mathrm{p} . \mathrm{u}$. \\
$k_{q}$ & Slope gain & $0.004 \Omega / \mathrm{V}$ \\
$k_{i}$ & Static integral gain & $787.78 \mathrm{~A} / \mathrm{s}$ \\
\hline
\end{tabular}

point digital signal processor was used as control platform. The current reference is generated following (1). Proportionalresonant controllers are used in the inner current loops [43]. The experimental setup is shown in Fig. 7.

The nominal values of the system parameters and design specifications are listed in Table I. $Q_{\max }$ is calculated using the maximum active power which can generate the power source and the rating of the converter. Then, in nominal conditions, it is desired a reactive power injection of $Q_{\max } / 2=500 \mathrm{VAr}$. The cross-over frequency is selected as $\omega_{c}=2 \pi \mathrm{rad} / \mathrm{s}$, which is more than one decade below the grid frequency and three decades below to the current loop bandwidth $(\approx 2 \pi 1300 \mathrm{rad} / \mathrm{s})$. According to this value of $\omega_{c}$, it is expected a settling time of approximately $t_{s}=0.8 \mathrm{~s}$.

In the first part, experimental results of the constant reactive power reference method and the constant voltage regulation method are presented. Then, the static slope voltage is analyzed under different grid voltages and different grid impedances. Next, the proposed adaptive slope voltage control is tested. Finally an analysis of the impact of resistive grid impedances over the dynamic properties of the proposal is performed.

All the tests start with the control strategies disabled, to appreciate the voltage imposed by the grid. At $t=0.4 \mathrm{~s}$, the control is activated leading to a transient response until steady-state operation values are reached. When it is the case, the impedance estimation method in [39] is also activated at $t=0.4 \mathrm{~s}$. The control parameters $k_{i}, V^{*}$ and $k_{q}$ are calculated from (19), (23) and (25). In some tests, a slight ripple in the reactive power is noticed. However. since its peak-to-peak value corresponds to less than $1 \%$ of the nominal reactive power, it is considered negligible over the static and dynamic properties of the system.

\section{A. Constant Reactive Power Reference}

First, the constant reactive power reference control was implemented. Three scenarios of $V_{g}$ have been programmed in the AC source in order to verify the system operation: $V_{g}=1.018$ p.u. represented with the red line, a nominal 

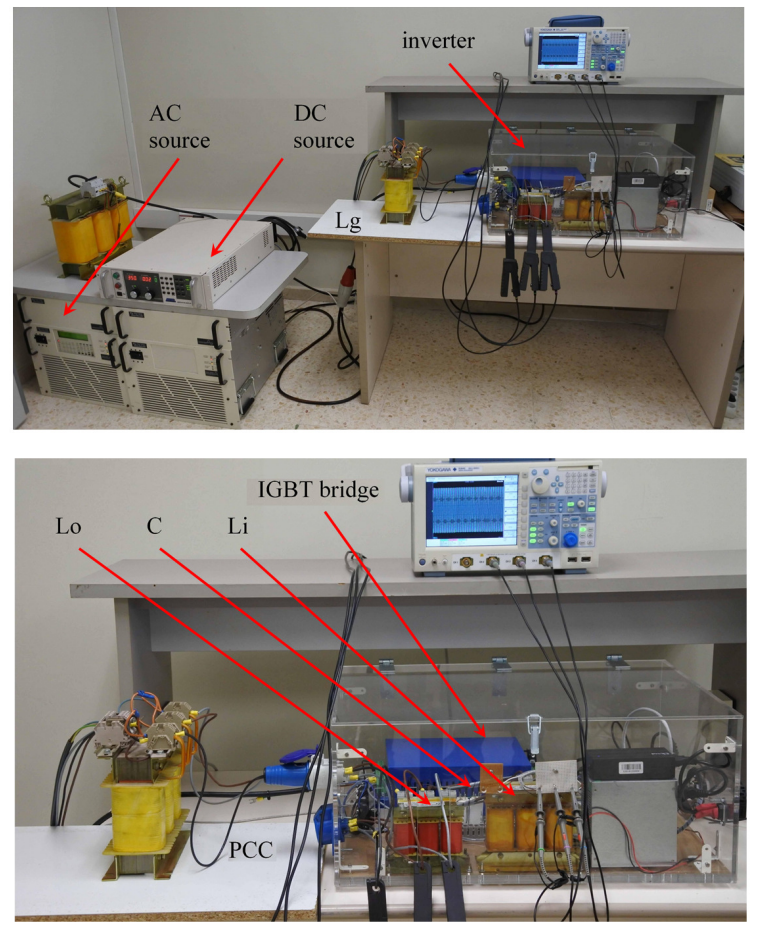

Figure 7. Experimental setup.

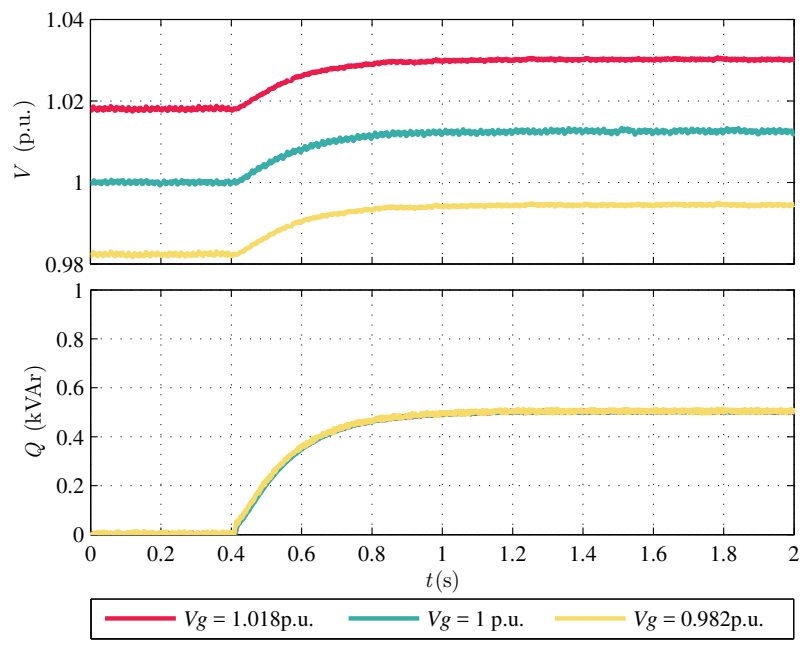

Figure 8. Transient response of the constant reactive power reference control for different grid voltage $V_{g}$ values. Top: output voltage. Bottom: reactive power.

value of $V_{g}=1$ p.u. represented with the blue line and $V_{g}=0.982$ p.u. represented with the yellow line. A constant value of grid inductance $\left(L_{g_{0}}=2.5 \mathrm{mH}\right)$ was used in all the cases. For this test, a reactive power reference $Q^{*}=500 \mathrm{VAr}$ was selected.

The experimental results are presented in Fig. 8. As it can be seen, in the three cases the injected reactive powers follow the reference in an accurate manner. The fixed reactive power reference produces a proportional increase in the PCC voltage, which can lead to undesired operation points according to the value of $V_{g}$. For this particular case, the scenario represented with a red line ( $V_{g}=1.018$ p.u.) suffers a voltage increase

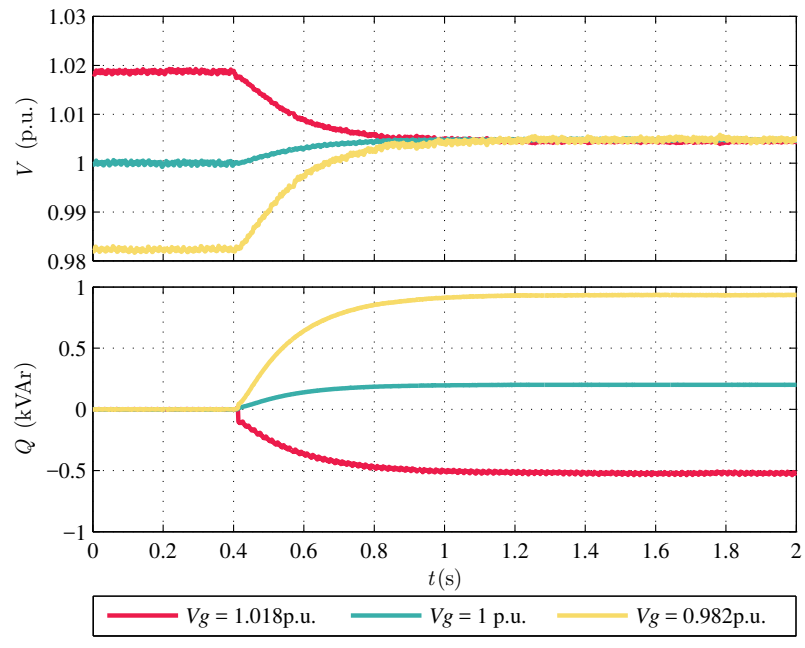

Figure 9. Transient response of the constant voltage regulation control for different grid voltage $V_{g}$ values. Top: output voltage. Bottom: reactive power.

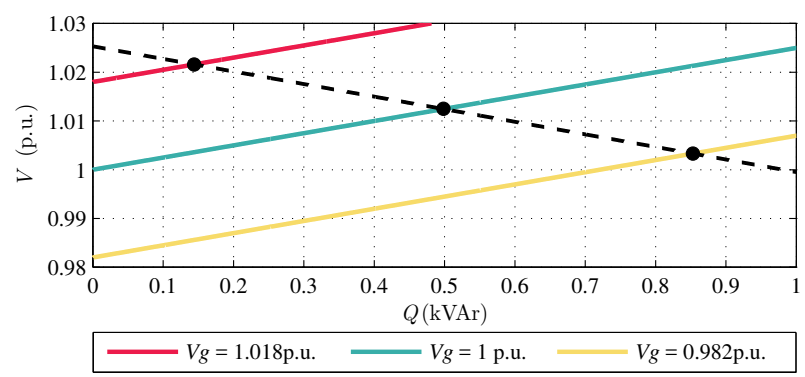

Figure 10. Performance of the static slope voltage control for different grid voltage $V_{g}$ values. Static diagram.

which leads the operation point at the limit of the desired boundaries (1.03 p.u.).

\section{B. Constant Voltage Regulation}

Next, the constant voltage regulation was implemented. The same $V_{g}$ scenarios of the previous test were considered, as well as the value of the grid inductance. For this test, a voltage reference $V^{*}=1.005$ p.u was selected.

The experimental results are presented in Fig. 9. As discussed in subsection II-B, usually this technique requires a large range of reactive power to satisfy the voltage objective, which can lead to operational points close to the maximum reactive power capacity (as is the case of the scenario represented with yellow line, $V_{g}=0.982$ p.u.) or, in some cases, to the impossibility of reaching the control objectives.

\section{Static Slope Voltage Control}

Next, a test using the static slope voltage control was performed. Again, the same $V_{g}$ scenarios and grid inductance were considered. Fig. 10 presents the modeling of the system performance. The diagram allows to appreciate the expected operation points.

The experimental results of the transient responses are shown in Fig. 11. The blue line $\left(V_{g}=1\right.$ p.u. $)$ corresponds to a operation point with a reactive power injection of 


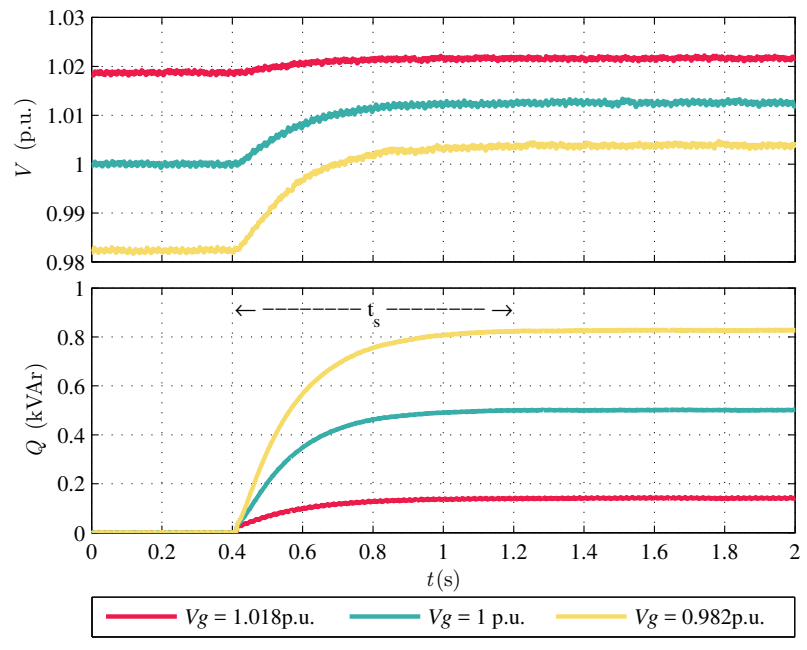

Figure 11. Transient response of the static slope voltage control for different grid voltage $V_{g}$ values. Top: output voltage. Bottom: reactive power.

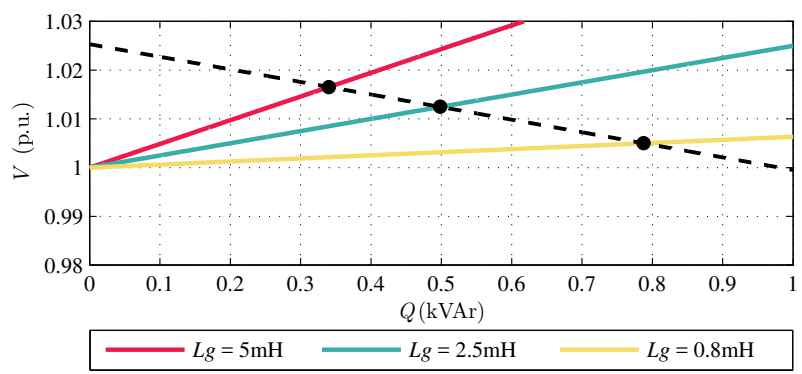

Figure 12. Performance of the static slope voltage control for different $L_{g}$. Static diagram.

$Q=500 \mathrm{VAr}$, as determined in the design procedure. A PCC voltage of 1.0125 p.u. is obtained. For the other implemented values of $V_{g}$ the control law dictates a reduction or an increase of the reactive power injection according to the grid voltage. Particularly, for the red line $\left(V_{g}=1.018\right.$ p.u. $)$ an operation point with $V=1.022$ p.u. and $Q=150 \mathrm{VAr}$ is obtained. In the other case, for the yellow line $\left(V_{g}=0.982\right.$ p.u. $)$ an operation point with $V=1.003$ p.u. and $Q=850 \mathrm{VAr}$ is obtained. Experimental results presented in Fig. 11 coincide precisely with the expected operation points, showing the accuracy of the system modeling.

In all tests, the settling time is approximately $0.8 \mathrm{~s}$, as expected according to the fixed $\omega_{c}$. This fact shows that a desired dynamic response can be guaranteed regardless the PCC voltage amplitude.

\section{Static Slope Voltage Control Under Different Grid Impedances}

In the next test, the static slope voltage control was evaluated under different grid impedances. Three inductances $L_{g}$ were tested: $0.8 \mathrm{mH}, 2.5 \mathrm{mH}$, and $5 \mathrm{mH}$. Similarly to the previous test, Fig. 12 and 13 present the modeling of the system performance and the experimental results of the transient responses. The design of the control parameters was done using the nominal grid impendance, i.e., $L_{g_{0}}=2.5 \mathrm{mH}$.

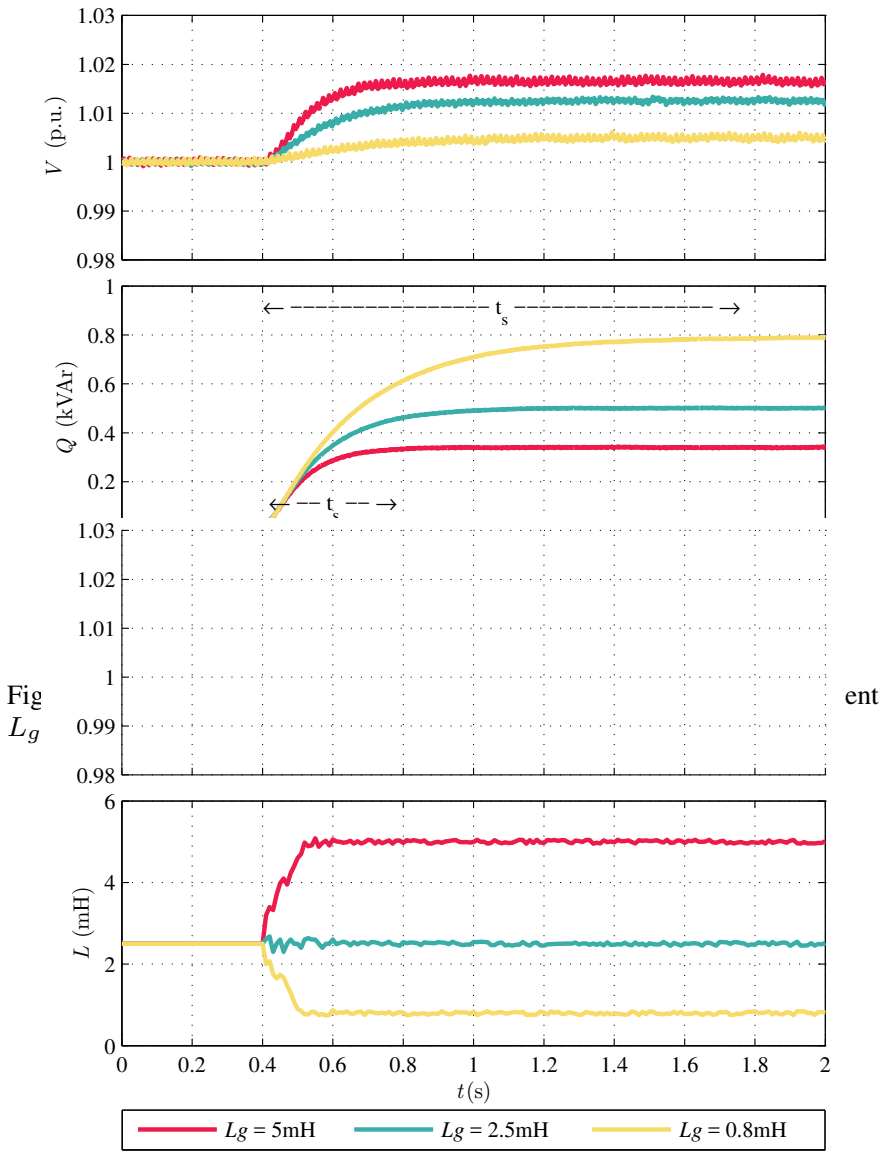

Figure 14. Estimated values of the grid impedance using the technique presented in [39].

Fig. 12 shows the operation points. Variations on the impedance produce different slopes in the system performance without affecting the control law. For $L_{g}=5 \mathrm{mH}$, an operation point with $V=1.017$ p.u. and $Q=350 \mathrm{VAr}$ is obtained while for $L_{g}=0.8 \mathrm{mH}$, the operation point achieved is $V=1.005$ p.u. and $Q=800$ VAr. Regarding the nominal impedance, $L_{g}=2.5 \mathrm{mH}$, the same operation point of previous test was achieved. The modeling of these operation points shows how an increase in the value of $L_{g}$ produces an increase in the PCC voltage with a corresponding reduction in the reactive power injection.

Fig. 13 shows the transient responses. Due to the changes in the grid impedance, variations on the settling time are observed. Particularly, with $L_{g}=5 \mathrm{mH}$ and $L_{g}=0.8 \mathrm{mH}$ the settling time are approximately $0.4 \mathrm{~s}$ and $1.3 \mathrm{~s}$, respectively. These values agree with the theoretical ones calculated using (20). Note that the variations of $L_{g}$ affect the time response of the control strategy, which corresponds to an undesired dynamic performance.

\section{E. Proposed Adaptive Slope Voltage Control}

In the last test, the proposed adaptive slope voltage control was evaluated. As explained in Section IV, the value of the parameter $k_{i}^{\prime}$ was updated according to (19) using a grid impedance estimation technique [39]. In Fig. 14 the estimated values are presented. The same inductances $L_{g}$ of the previous test were implemented $(0.8 \mathrm{mH}, 2.5 \mathrm{mH}$ and $5 \mathrm{mH})$. 


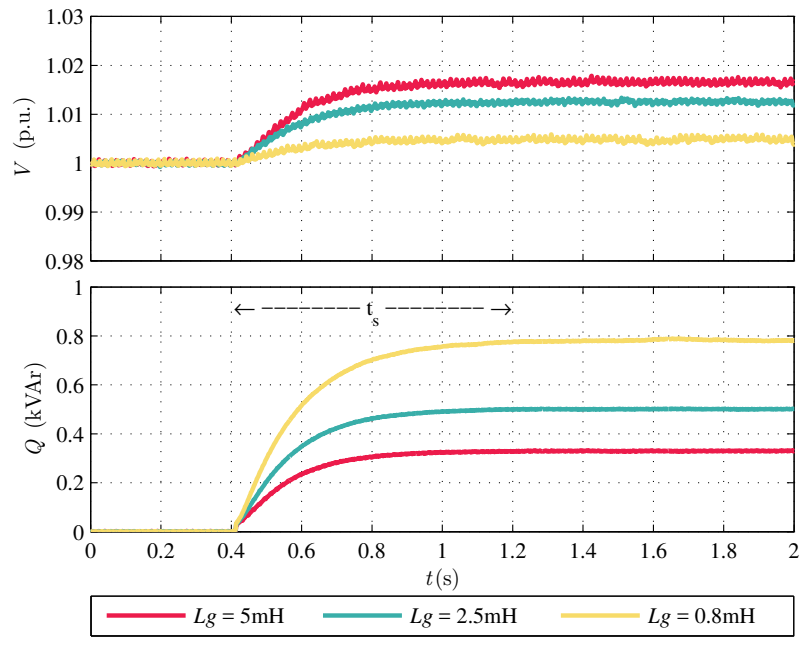

Figure 15. Transient response of the adaptive slope voltage control for different $L_{g}$ values. Top: output voltage. Bottom: reactive power.

Fig. 15 presents the transient responses. The results show how the adaptive control allows to obtain a settling time of $0.8 \mathrm{~s}$ in all the tests. The operation point values are equal to those obtained in the previous test, considering that the parameters $k_{q}$ and $V^{*}$ were not modified. Note that the effect on the system dynamics produced by the transient in the grid impedance estimation is almost negligible. The main reason is that this transient is seen as a high frequency noise which is rejected by the behavior of the low pass filter expressed in (16).

In this way, the proposed control overcomes the issues related to the dynamic response that the changes in the grid impedance can generate. The proposed solution not only is characterized by its simplicity, but also for its accurate operation performance.

\section{F. Impact of Resistive Grid Impedance over Dynamic Proper- ties}

As discussed in Section II, the modeling presented in this paper was based on the assumption that the grid impedances are mostly inductive. However, this may not be applicable in all cases for distributed systems. Considering this, the impact of resistive grid impedances over the dynamic properties of the proposal is analyzed in this section.

As can be seen in the previous experimental results, the adaptive characteristic proposed in this paper provides the slope control with robustness against variations in the grid impedance, which translate into the possibility of ensuring a constant settling time. Thus, to analyze the impact that the resistive component of the grid impedance may have on this characteristic, different combinations of resistance and inductance were tested to measure the settling time changes. The values are listed in Table II, in which is also present the $R_{g} / X_{g}$ ratio.

The experimental results are shown in Fig.16. On it, different values of $R_{g} / X_{g}$ (depicted in colors according the value of $L_{g}$ ) are contrasted with their corresponding settling times. The discontinuous line emphasizes the proportional relationship
Table II

CONSIDERED VALUES FOR THE GRID IMPEDANCE

\begin{tabular}{ccc}
\hline \hline$L_{g}$ & $R_{g}$ & Ratio $R_{g} / X_{g}$ \\
\hline $0.8 \mathrm{mH}$ & $0.25 \Omega$ & 0.8289 \\
\hline $2.5 \mathrm{mH}$ & $0.25 \Omega$ & 0.2653 \\
$2.5 \mathrm{mH}$ & $0.5 \Omega$ & 0.5305 \\
$2.5 \mathrm{mH}$ & $1 \Omega$ & 1.061 \\
\hline $5 \mathrm{mH}$ & $0.25 \Omega$ & 0.1326 \\
$5 \mathrm{mH}$ & $0.5 \Omega$ & 0.2653 \\
$5 \mathrm{mH}$ & $1 \Omega$ & 0.5305 \\
$5 \mathrm{mH}$ & $1.5 \Omega$ & 0.7958 \\
\hline
\end{tabular}

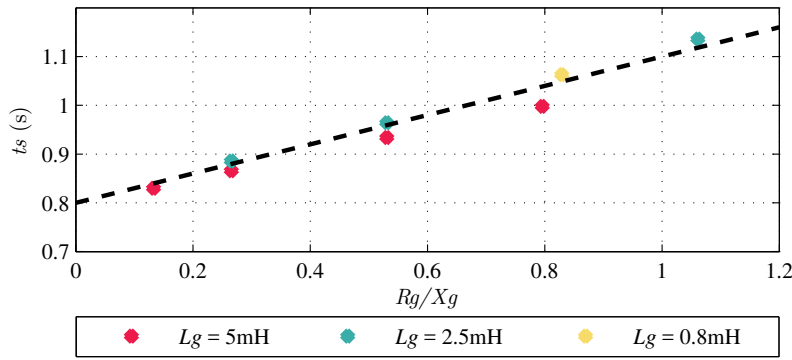

Figure 16. $R_{g} / X_{g}$ grid impedance ratio vs. settling time of the proposed control.

that $R_{g} / X_{g}$ has with the response time. If a maximum of $15 \%$ variation in settling time is is allowed, then the proposal can work correctly in scenarios with $R_{g} / X_{g}$ ratios of up to 0.4. Higher values cause an unacceptable increase in the response time.

\section{Conclusions}

This paper presents an adaptive slope voltage control based on a real-time estimation of the equivalent grid impedance. The proposal offers an excellent transient performance against operating variations, guaranteeing a constant settling time response without affecting the steady-state operation point. The main expressions of the proposal are derived from a controloriented mathematical model which are also used to present design considerations for the control parameters. Experimental results are reported, confirming the accuracy of the modeling and the performance of the proposal.

\section{APPENDIX}

This appendix gives further details of the simplified averaged model of the grid-connected DG.

\section{A. Averaged Model of the Grid-Connected DG}

The averaging approach is based on a harmonic balance in which the large-signal model is approximated considering the slow variations of the amplitudes of the sinusoidal signals. In this way, the model can be written in a general form as

$$
f_{1 c} \cos \omega t+f_{1 s} \sin \omega t=f_{2 c} \cos \omega t+f_{2 s} \sin \omega t .
$$

From (A1), the averaged model can be derived by equaling the amplitudes as 


$$
\begin{aligned}
& f_{1 c}=f_{2 c} \\
& f_{1 s}=f_{2 s} .
\end{aligned}
$$

By inserting (4), (5) and (6) in (3), the large-signal model general form is obtained, where

$$
\begin{gathered}
f_{1 c}=V_{g} \cos \phi \\
f_{1 s}=V_{g} \sin \phi \\
f_{2 c}=V-\frac{2 L_{g}}{3 V}\left(Q^{*} \omega+\frac{d P^{*}}{d t}-\frac{P^{*}}{V} \frac{d V}{d t}\right) \\
f_{2 s}=\frac{2 L_{g}}{3 V}\left(\frac{d Q^{*}}{d t}-P^{*} \omega-\frac{Q^{*}}{V} \frac{d V}{d t}\right) .
\end{gathered}
$$

Then, applying harmonic balance, the average large-signal model is obtained as

$$
\begin{aligned}
V_{g}^{2}=( & \left.V-\frac{2 L_{g}}{3 V}\left(Q^{*} \omega+\frac{d P^{*}}{d t}-\frac{P^{*}}{V} \frac{d V}{d t}\right)\right)^{2} \\
+ & \left(\frac{2 L_{g}}{3 V}\left(\frac{d Q^{*}}{d t}-P^{*} \omega-\frac{Q^{*}}{V} \frac{d V}{d t}\right)\right)^{2}
\end{aligned}
$$

Notice that the right-hand side of (A8) is composed by two terms. The first of them includes the amplitude $V$ which makes this term considerable higher than the second one. For this reason, neglecting the second term in front of the first term can be considered as an appropriate assumption. Thus, the simplified model can be rewritten as is expressed in (7).

\section{REFERENCES}

[1] T. Adefarati and R. C. Bansal, "Integration of renewable distributed generators into the distribution system: a review," IET Renewable Power Generation, vol. 10, no. 7, pp. 873-884, 2016.

[2] F. Blaabjerg, R. Teodorescu, M. Liserre, and A. V. Timbus, "Overview of control and grid synchronization for distributed power generation systems," IEEE Trans. Ind. Electron., vol. 53, no. 5, pp. 1398-1409, Oct 2006.

[3] N. K. Roy and H. R. Pota, "Current status and issues of concern for the integration of distributed generation into electricity networks," IEEE Systems Journal, vol. 9, no. 3, pp. 933-944, Sept. 2015.

[4] J. A. P. Lopes, C. L. Moreira, and A. G. Madureira, "Defining control strategies for microgrids islanded operation," IEEE Trans. on Power Systems, vol. 21, no. 2, pp. 916-924, 2006.

[5] J. Rocabert, A. Luna, F. Blaabjerg, and P. Rodríguez, "Control of power converters in AC microgrids," IEEE Trans. Power Electron., vol. 27, no. 11, pp. 4734-4749, Nov 2012.

[6] J. M. Guerrero, J. L. G. de Vicuña, J. Matas, M. Castilla, and J. Miret, "A wireless controller to enhance dynamic performance of parallel inverters in distributed generation systems," IEEE Trans. on Power Electro., vol. 19, no. 5, pp. 1205-1213, Sept 2004.

[7] J. M. Guerrero, J. C. Vasquez, J. Matas, J. L. G. de Vicuña, and M. Castilla, "Hierarchical control of droop-controlled AC and DC microgrids - A general approach toward standardization," IEEE Trans. on Industrial Electronics, vol. 58, no. 1, pp. 158-172, Jan 2011.

[8] J. M. Guerrero, J. C. Vasquez, J. Matas, L. García De Vicuña, and M. Castilla, "Hierarchical control of droop-controlled AC and DC microgrids-A general approach toward standardization," IEEE Trans. Ind. Electronics, vol. 58, no. 1, pp. 158-172, Jan 2011.

[9] J. L. Sosa, M. Castilla, J. Miret, J. Matas, and Y. A. Al-Turki, "Control strategy to maximize the power capability of PV three-phase inverters during voltage sags," IEEE Trans. Power Electron., vol. 31, no. 4, pp. 3314-3323, April 2016.
[10] C. T. Lee, C. W. Hsu, and P. T. Cheng, "A low-voltage ride-through technique for grid-connected converters of distributed energy resources," IEEE Trans. Ind. Appl., vol. 47, no. 4, pp. 1821-1832, July 2011.

[11] J. Miret, A. Camacho, M. Castilla, J. L. G. de Vicuña, and J. de la Hoz, "Reactive current injection protocol for low-power rating distributed generation sources under voltage sags," IET Power Electron., vol. 8, no. 6 , pp. 879-886, June 2015.

[12] T. Ackermann, Wind power in power systems. John Wiley \& Sons, 2005.

[13] M. Tsili and S. Papathanassiou, "A review of grid code technical requirements for wind farms," IET Renewable Power Generation, vol. 3, no. 3, pp. 308-332, Sep. 2009.

[14] J. Martínez, P. C. Kjaer, P. Rodriguez, and R. Teodorescu, "Comparison of two voltage control strategies for a wind power plant," in 2011 IEEE/PES Power Systems Conference and Exposition, March 2011, pp. $1-9$.

[15] J. Martinez and P. Kjaer, "Fast voltage control in wind power plants," in Power and Energy Society General Meeting, IEEE, Jul. 2011, pp. 1-7.

[16] J. Martínez, P. C. Kjr, P. Rodriguez, and R. Teodorescu, "Design and analysis of a slope voltage control for a DFIG wind power plant," IEEE Trans. Energy Convers., vol. 27, no. 1, pp. 11-20, March 2012.

[17] "IEEE recommended practice for excitation system models for power system stability studies," IEEE Std 421.5-2016 (Revision of IEEE Std 421.5-2005), pp. 1-207, Aug 2016.

[18] A. Bidram and A. Davoudi, "Hierarchical structure of microgrids control system," IEEE Trans. on Smart Grid, vol. 3, no. 4, pp. 1963-1976, Dec 2012.

[19] J. Kim, J. Seok, E. Muljadi, and Y. C. Kang, "Adaptive QV scheme for the voltage control of a DFIG-based wind power plant," IEEE Trans. on Power Electronics, vol. 31, no. 5, pp. 3586-3599, May 2016.

[20] L. Petersen, F. Kryezi, and F. Iov, "Design and tuning of wind power plant voltage controller with embedded application of wind turbines and STATCOMs," IET Renewable Power Generation, vol. 11, no. 3, pp. 216 225, 2017.

[21] M. Castilla, J. Miret, A. Camacho, J. L. G. de Vicuña, and J. Matas, "Modeling and design of voltage support control schemes for threephase inverters operating under unbalanced grid conditions," IEEE Trans. on Power Electron., vol. 29, no. 11, pp. 6139-6150, Jan. 2014.

[22] J. Torres-Martínez, M. Castilla, J. Miret, M. Moradi-Ghahderijani, J. Morales, and R. Guzman, "Dynamic model of a grid-connected threephase inverter with slope voltage control," in Industrial Electronics Society, IECON 2015 - 41st Annual Conference of the IEEE, Nov 2015, pp. $1228-1233$.

[23] E. Figueres, G. Garcera, J. Sandia, F. Gonzalez-Espin, and J. C. Rubio, "Sensitivity study of the dynamics of three-phase photovoltaic inverters with an LCL grid filter," IEEE Trans. Ind. Electron., vol. 56, no. 3, pp. 706-717, Nov. 2009.

[24] IEEE Application Guide for IEEE Std 1547, IEEE Standard for Interconnecting Distributed Resources with Electric Power Systems, IEEE Standard 1547.2-2008, 2009.

[25] M. Altin, Ö. Göksu, R. Teodorescu, P. Rodriguez, B. B. Jensen, and L. Helle, "Overview of recent grid codes for wind power integration," in 2010 12th International Conference on Optimization of Electrical and Electronic Equipment, May 2010, pp. 1152-1160.

[26] P. Rodriguez, A. V. Timbus, R. Teodorescu, M. Liserre, and F. Blaagjerg, "Independent PQ control for distributed power generation systems under grid faults," in IECON 2006 - 32nd Annual Conference on IEEE Industrial Electronics, Nov 2006, pp. 5185-5190.

[27] P. Rodriguez, A. V. Timbus, R. Teodorescu, M. Liserre, and F. Blaabjerg, "Flexible active power control of distributed power generation systems during grid faults," IEEE Trans. Ind. Electron., vol. 54, no. 5, pp. 2583 2592, Oct 2007

[28] P. Rodriguez, A. Luna, R. S. Muñoz Aguilar, I. Etxeberria-Otadui, R. Teodorescu, and F. Blaabjerg, "A stationary reference frame grid synchronization system for three-phase grid-connected power converters under adverse grid conditions," IEEE Trans. Power Electron., vol. 27, no. 1, pp. 99-112, Jan. 2012.

[29] M. Triggianese, J. Morren, S. W. H. de Haan, and P. Marino, "Improved and extended DG capability in voltage regulation by reactive and active power," in 2007 International Conference on Power Engineering, Energy and Electrical Drives, April 2007, pp. 583-588.

[30] M. Castilla, A. Camacho, P. Martí, M. Velasco, and M. M. Ghahderijani, "Impact of clock drifts on communication-free secondary control schemes for inverter-based islanded microgrids," IEEE Trans. Ind. Electron., vol. 65, no. 6, pp. 4739-4749, June 2018.

[31] M. Andreasson, D. V. Dimarogonas, H. Sandberg, and K. H. Johansson, "Distributed control of networked dynamical systems: Static feedback, 
integral action and consensus," IEEE Trans. on Automatic Control, vol. 59, no. 7, pp. 1750-1764, July 2014.

[32] K. Mahabir, G. Verghese, J. Thottuvelil, and A. Heyman, "Linear averaged and sampled data models for large signal control of high power factor ac-dc converters," in Power Electronics Specialists Conference, 1990. PESC '90 Record., 21st Annual IEEE, 1990, pp. 372-381.

[33] M. Liserre, R. Teodorescu, and F. Blaabjerg, "Stability of photovoltaic and wind turbine grid-connected inverters for a large set of grid impedance values," IEEE Trans. Power Electron., vol. 21, no. 1, pp. 263-272, Jan 2006.

[34] A. Vidal, A. G. Yepes, F. D. Freijedo, O. López, J. Malvar, F. Baneira, and J. Doval-Gandoy, "A method for identification of the equivalent inductance and resistance in the plant model of current-controlled gridtied converters," IEEE Trans. Power Electron., vol. 30, no. 12, pp. 72457261, Dec 2015.

[35] M. Liserre, F. Blaabjerg, and R. Teodorescu, "Grid impedance estimation via excitation of LCL-filter resonance," IEEE Trans. Ind. Appl., vol. 43, no. 5, pp. 1401-1407, Sept 2007.

[36] A. Ghanem, M. Rashed, M. Sumner, M. A. Elsayes, and I. I. I. Mansy, "Grid impedance estimation for islanding detection and adaptive control of converters," IET Power Electron., vol. 10, no. 11, pp. 1279-1288, 2017.

[37] N. Hoffmann and F. W. Fuchs, "Minimal invasive equivalent grid impedance estimation in inductive - resistive power networks using extended Kalman filter," IEEE Trans. Power Electron., vol. 29, no. 2, pp. 631-641, Feb 2014.

[38] L. Asiminoaei, R. Teodorescu, F. Blaabjerg, and U. Borup, "Implementation and test of an online embedded grid impedance estimation technique for PV inverters," IEEE Trans. Ind. Electron., vol. 52, no. 4, pp. 1136 - 1144, Aug. 2005

[39] S. Cobreces, E. Bueno, D. Pizarro, F. Rodriguez, and F. Huerta, "Grid impedance monitoring system for distributed power generation electronic interfaces," IEEE Trans. Instrum. Meas., vol. 58, no. 9, pp. 3112 - 3121, Sep. 2009.

[40] K. Lin, F. Xiao, and G. Jie, "Grid impedance estimation based on particle swarm optimization," in 2017 IEEE 2nd Advanced Information Technology, Electronic and Automation Control Conference (IAEAC), March 2017, pp. 573-576.

[41] S. Cobreces, F. Huerta, D. Pizarro, F. J. Rodrguez, and E. J. Bueno, "Three-phase power system parametric identification based on complexspace recursive least squares," in 2007 IEEE International Symposium on Intelligent Signal Processing, Oct 2007, pp. 1-6.

[42] S. Cobreces, P. Rodriguez, D. Pizarro, F. J. Rodriguez, and E. J. Bueno, "Complex-space recursive least squares power system identification," in 2007 IEEE Power Electronics Specialists Conference, June 2007, pp. $2478-2484$.

[43] D. N. Zmood, D. G. Holmes, and G. H. Bode, "Frequency-domain analysis of three-phase linear current regulators," IEEE Trans. Ind. Appl., vol. 37, no. 2, pp. 601-610, Mar./Apr. 2001.

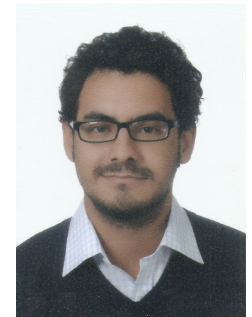

Juan M. Rey was born in Bucaramanga, Colombia in 1989. He received the B.S. in electrical engineering from Universidad Industrial de Santander, Bucaramanga, Colombia, in 2012. He is currently working toward the Ph.D. degree in the Department of Electronic Engineering, Technical University of Catalonia, Spain. Since 2013, he has been with the Electrical, Electronic and Telecommunications Engineering School (E3T), Universidad Industrial de Santander, Bucaramanga Colombia, where he is currently an Assistant Professor. His research interest are power electronics and control for distributed generation and microgrids.

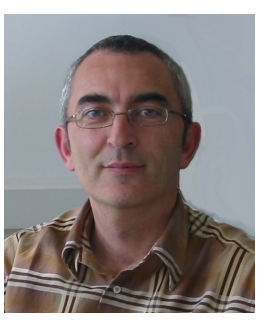

Miguel Castilla received the B.S., M.S., and Ph.D. degrees in telecommunication engineering from the Technical University of Catalonia, Barcelona, Spain, in 1988 , 1995, and 1998, respectively. Since 2002, he has been an Associate Professor with the Department of Electronic Engineering, Technical University of Catalonia, where he teaches courses on analog circuits and power electronics. His research interests include power electronics, nonlinear control, and renewable energy systems.

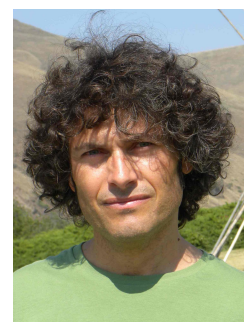

Jaume Miret (M98) received the B.S. degree in telecommunications, the M.S. degree in electronics, and the Ph.D. degree in electronics from the Universitat Politecnica de Catalunya, Barcelona, Spain, in 1992, 1999, and 2005, respectively. From 1993 to 2011, he was an Assistant Professor with the Department of Electronic Engineering, Universitat Politecnica de Catalunya, where since 2011, he has been an Associate Professor and teaches courses on digital design and circuit theory. His research interests include dc-to-ac converters, active power filters, and digital control.

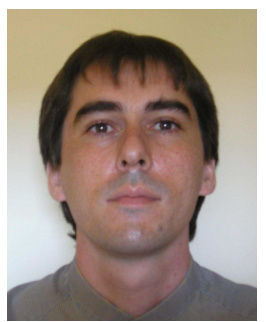

Antonio Camacho received the B.S. degree in chemical engineering, the M.S. degree in automation and industrial electronics, and the Ph.D. degree in electronic engineering, from the Technical University of Catalonia, Barcelona, Spain in 2000, 2009 and 2015 respectively. His research interests include networked and embedded control systems, industrial informatics, and power electronics.

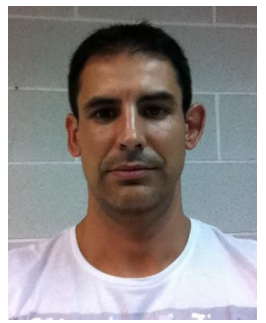

Ramon Guzman received the B.S., the M.S. and the $\mathrm{Ph} . \mathrm{D}$. degrees in telecommunications engineering from the Technical University of Catalonia, Barcelona, Spain, in 1999, 2004 and 2016, respectively. He is currently an Associate Professor with the Department of Automatic Control in the Technical University of Catalonia. His research interests include nonlinear and adaptive control for threephase power converters. 